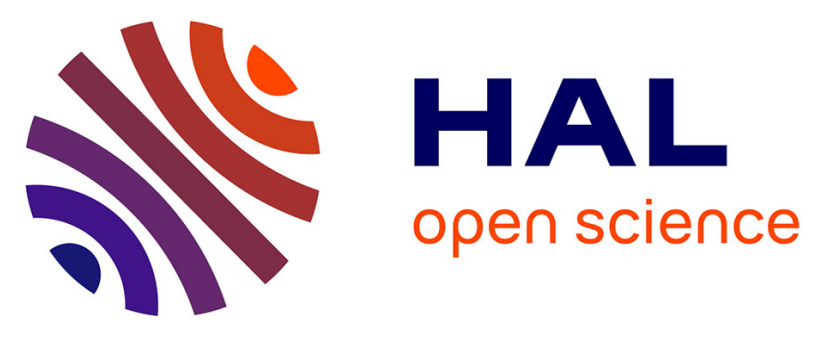

\title{
Les représentations de la drépanocytose comme déterminants de l'observance thérapeutique
}

Damien Oudin-Doglioni, Marie-Claire Gay, Marie-Pierre Lehougre, Jean-Benoît Arlet, Frédéric Galactéros

\section{- To cite this version:}

Damien Oudin-Doglioni, Marie-Claire Gay, Marie-Pierre Lehougre, Jean-Benoît Arlet, Frédéric Galactéros. Les représentations de la drépanocytose comme déterminants de l'observance thérapeutique. Annales Médico-Psychologiques, Revue Psychiatrique, 2019, 177, pp.517 - 525. 10.1016/j.amp.2017.10.024 . hal-03484380

\section{HAL Id: hal-03484380 \\ https://hal.science/hal-03484380}

Submitted on 20 Dec 2021

HAL is a multi-disciplinary open access archive for the deposit and dissemination of scientific research documents, whether they are published or not. The documents may come from teaching and research institutions in France or abroad, or from public or private research centers.
L'archive ouverte pluridisciplinaire HAL, est destinée au dépôt et à la diffusion de documents scientifiques de niveau recherche, publiés ou non, émanant des établissements d'enseignement et de recherche français ou étrangers, des laboratoires publics ou privés.

\section{(ㄷ)(1) $\$$}

Distributed under a Creative Commons Attribution - NonCommerciall 4.0 International 


\section{Mémoire}

Les représentations de la drépanocytose comme déterminants de l'observance thérapeutique

\section{Sickle cell disease representation as a determinant for patient compliance} Damien Oudin-Doglioni a ${ }^{\text {, Marie-Claire Gay }}{ }^{\text {b }}$, Marie-Pierre Lehougre ${ }^{\text {c }}$, JeanBenoît Arlet ${ }^{d}$, Frédéric Galactéros ${ }^{e}$

a) Damien Oudin-Doglioni, Université Paris Nanterre, 92000 Nanterre, France

b) Pr Marie-Claire Gay, CLIPSYD-EA4430 (ED139CLM), Université Paris Nanterre, 92000 Nanterre, France

c) Marie-Pierre Lehougre, Psychologue clinicien, Unité des Maladies Génétiques du Globule Rouge, Hôpital Henri Mondor, 94000 Créteil, France

d) Dr Jean-Benoît Arlet, MCU-PH, Service de Médecine Interne - Hôpital Européen George Pompidou, 75015 Paris,

e) Pr Frédéric Galactéros, PU-PH, Chef de service, Unité des Maladies Génétiques du Globule Rouge - Hôpital Henri Mondor, 94000 Créteil, France

\section{Texte reçu le 22 août ; accepté le 12 octobre 2017}

\section{Résumé}

La drépanocytose est la plus fréquente des maladies génétiques graves au monde et en France. Malgré l'amélioration que confère la prise en charge médicale actuelle, le taux de non-adhésion aux traitements reste fort, conduisant au maintien d'un niveau d'hospitalisation élevé. Dans d'autres maladies chroniques, un lien a été montré entre la représentation de la maladie (selon le modèle de Leventhal) et l'observance thérapeutique. Cependant, ce lien n'a pas été montré dans une maladie génétique et aucune donnée n'existe sur la drépanocytose. Dans ce travail, nous avons tenté une première approche des dimensions de la représentation qui pourraient influencer l'observance et l'adhésion thérapeutique. Les analyses effectuées suggèrent un modèle à trois facteurs : la chronicité perçue de la maladie, la perception de l'efficacité des traitements et les émotions négatives associées à la maladie influencent l'observance thérapeutique et expliquent 29,2 \% de sa variance. Nos résultats montrent que le modèle de Leventhal peut s'appliquer dans 
une maladie chronique d'origine génétique dont l'étiologie est connue, permettant de mieux comprendre le rôle de la représentation de la maladie sur différentes dimensions, dont l'observance thérapeutique. Cette recherche exploratoire est un point de départ pour le développement d'autres études afin d'apporter une meilleure connaissance de cette maladie.

Mots clés : Adhésion au soin ; Drépanocytose ; Représentation de la maladie ; Observance

\begin{abstract}
Sickle Cell Disease (SCD) is a major health care and societal problem that affects millions people wordwild. In France, SCD is the most common genetic disorder. Although medical care is improving, non-compliance rates are still high leading to a high rate of hospitalization. In other chronic illnesses, compliance have been explained by dimensions of the illness representation (as defined in the Common Sense Model (CSM) of Leventhal). However no such work has been done in genetic disorder nor data have been collected in SCD. Here we show that illness representation influences compliance. Analyse showed that three main factors of representation might have an influence on compliance in SCD and explain 29,2\% of the variance. The emotional representation (emotional perception of SCD), treatment control (perceived effectiveness of treatments) and timeline acute/chronic (perceived duration of illness) indicating that patients with negative emotions on SCD are less compliant and those who perceive the effectiveness of their treatment and SCD as a chronic disease are more compliant. Compliance in SCD appears to be influenced by illness representations in the CSM. This give us a theoretical background to understand adaptation to the SCD and to propose psychosocial interventions aiming at a better compliance in people with SCD.
\end{abstract}

Keywords: Adherence; Compliance; Illness representation; Sickle cell disease

\title{
1. Introduction : brève présentation de la drépanocytose, de son épidémiologie et de ses implications psychologiques
}

Selon l'OMS [31] «près de $5 \%$ de la population mondiale portent les gènes caractéristiques des hémoglobinopathies, qui sont principalement ceux de la drépanocytose et de 
la thalassémie ». Le trait S serait porté par près de 120 millions d'individus dans le monde et environ 300000 enfants portant une hémoglobinopathie grave naissent chaque année dans le monde [33]. En France, elle est également la première maladie génétique ; ainsi, son taux dans les naissances était de 1/780 en 2010, en Île-de-France [7].

La drépanocytose est une maladie génétique de l'hémoglobine de transmission autosomique récessive. Cette protéine essentielle au transport de l'oxygène voit sa structure et ses propriétés modifiées par la mutation d'un seul acide aminé. Cette atteinte qualitative de sa structure aboutit à la formation de globules rouges falciformés et privés de leur propriété essentielle : la déformabilité [16]. Le trait drépanocytaire s'est maintenu à une prévalence de haut niveau par sélection favorable dans les pays impaludés du sud de la planète [19] et s'est diffusé par les mouvements de population (esclavage, colonisation et émigration) dans d'autres parties du monde. Plusieurs formes pathologiques existent : la plus répandue est la forme homozygote SS ; il existe également des formes hétérozygotes composites : SC, S $\beta$-thalassémie et SD Punjab [2].

Cette maladie se caractérise par de nombreux symptômes dont les plus fréquents sont : une anémie chronique par manque de globules rouges entraînant une asthénie et/ou une fatigabilité plus importante à l'effort, des crises de douleur aiguë par occlusion des vaisseaux sanguins à cause des globules rouges falciformés (crise vaso-occlusive osseuse, CVO) et une plus grande sensibilité aux infections (en particulier à germes encapsulés dont le pneumocoque) [23]. D'autres complications liées à l'occlusion des vaisseaux sanguins existent comme les syndromes thoraciques aigus (STA), les accidents vasculaires cérébraux (AVC) ou les nécroses osseuses, notamment de la hanche [11]. Selon les études françaises et anglo-saxonnes [21], le taux d'hospitalisation annuel des patients suivis est relativement stable, entre 25 et $30 \%$.

Les traitements proposés sont médicaux (e.g. échanges transfusionnels, allogreffe de moelle osseuse) et chimio-prophylactiques (e.g. hydroxyurée, supplémentation en acide folique, vaccination élargies, antibiothérapie). S'y ajoute un certain nombre de précautions dans le mode de vie (e.g. bonne hydratation, régulation de l'activité physique) [23]. Un bon suivi de ces traitements permet de réduire les «complications bruyantes » [23], notamment la douleur, par exemple, en limitant les occlusions vasculaires par l'augmentation du taux d'hémoglobine foetale (hydroxyurée) ou en réduisant la viscosité des globules rouges falciformés par l'amélioration du niveau d'hydratation cellulaire. Ces traitements permettent d'augmenter significativement l'espérance de vie [8]. On constate la forte diminution de la mortalité infantile qui permet à plus 
de $95 \%$ des enfants drépanocytaires d'atteindre l'âge adulte [23], et une augmentation significative de l'espérance de vie [28,12].

La drépanocytose est une maladie chronique avec des complications aiguës intriquées. Les premières crises vaso-occlusives pouvant apparaître dès quatre mois de vie et l'intrusion de la douleur et du monde médical dans la vie du sujet auront des conséquences possiblement importantes sur sa construction identitaire et son équilibre psychique. En effet, contrairement à des maladies chroniques acquises, comme la sclérose en plaque ou le VIH, les personnes atteintes par la drépanocytose se sont développées, physiquement et psychiquement, avec elle.

Les retentissements psychologiques proviennent essentiellement de la douleur et de l'impact de la maladie sur la vie quotidienne, notamment en termes de fatigue, mais aussi des représentations particulièrement catastrophiques qui entourent la maladie. Les effets de stigmatisation sont induits par les manifestations physiques de la maladie (e.g. œil jaune, fatigabilité confondu avec la paresse) auxquelles s'ajoutent la prédiction d'une mort précoce. Les troubles psychiques les plus fréquents sont la dépression (20,6\% [38]), l'anxiété (6,5\% [32]), et les conséquences psychosociales directement liées à la douleur telles que retrait social, agression, faible relation et désinvestissement scolaire [1].

Les traitements contre la symptomatologie drépanocytaire ont pour objectif de diminuer (e.g. grâce à un traitement par hydroxyurée) voire supprimer (e.g. après une allogreffe de moelle osseuse) les symptômes invalidants et de prévenir les complications graves et les hospitalisations. Hormis les traitements par thérapie cellulaire (i.e. allogreffe) ou actes médicaux (e.g. échanges transfusionnels), la posologie consiste souvent en une prise quotidienne per os. Malgré leur efficacité, notamment sur la survenue des crises douloureuses, les études rapportent un taux de non-observance variant de $37 \%$ à $71 \%[9,35,39]$.

\section{Aspects théoriques : représentation de la maladie et adhésion thérapeutique}

\subsection{L'observance et l'adhésion thérapeutique}

\subsubsection{Définition et problème de mesure}

L'ensemble des conduites amenant le patient à suivre les prescriptions d'un médecin a pris 
de nombreuses dénominations. Observance (compliance), adhésion (adherence) ou alliance thérapeutique (therapeutic alliance), montrant l'évolution d'une notion qui s'est dégagée progressivement de l'autorité médicale [3] : quand un patient est dit observant, il est sous-entendu la contrainte ; quand il est dit adhérent, on considère qu'il se conforme volontairement à des normes ou des standards établis pour lui. Dans la continuité de Barofsky [3], Haynes et al. [13] définissent l'adhésion thérapeutique comme le degré d'accord entre le comportement du patient et les conseils médicaux ou sanitaires.

S'il peut être théoriquement construit, le comportement d'adhésion thérapeutique reste difficilement mesurable compte tenu de sa nature multifactorielle et des nombreux biais de mesures qu'il suppose (e.g. désirabilité sociale). Il en existe des mesures objectives ou directes reposant sur le suivi et/ou le contrôle des patients à partir de marqueurs biologiques, ou bien des mesures subjectives ou indirectes se fondant sur l'auto-report ou l'hétéro-report. Ces types de mesures subjectives ou indirectes peuvent être inappropriés puisqu'elles n'indiquent pas les raisons pour lesquelles le patient ne suit pas ses prescriptions [24,25,36,37]. Néanmoins, ces mesures indirectes, quoique moins objectives, restent plus faciles à mettre en place. Elles consistent à opérationaliser une variable latente, car inobservable, grâce au score d'un questionnaire: les croyances sur les médicaments ou le comportement de prise de ses médicaments.

Afin de valider le lien entre les croyances spécifiques sur leur traitement et le comportement consistant à le suivre, Horne et Weinman ont comparé les résultats obtenus à leur questionnaire sur les croyances (Beliefs about Medecines Questionnaire, BMQ) avec une échelle auto-rapportée d'observance construite par Morisky et al. [26] : Morisky Medication Adherence Scale (MMAS) (validation française de 2012 par Korb-Savoldelli et al. [17]. Les résultats sont significatifs, et en particulier, la différence Nécessité-Préoccupations est un bon prédicteur de l'adhésion thérapeutique. Pour cette étude, la confirmation de cette corrélation entre les deux outils psychométriques (BMQ et MMAS) dans une population adulte atteinte par la drépanocytose sera effectuée.

\subsubsection{Non-observance et représentation de la drépanocytose}

La non-observance dans le cas de la drépanocytose a pour conséquence des 
hospitalisations pour le traitement des infections ou de la douleur. Une étude comparative entre la Guadeloupe et l'Angleterre sur les motifs d'hospitalisation entre 1987 et 2000 (crises douloureuses osseuses et/ou abdominales) et leurs facteurs déclenchants (e.g. pratique sportive excessive, absorption d'alcool, froid, manque d'hydratation), montre qu'ils apparaissent à la fois identiques et statistiquement stables dans leur répartition [21].

Par ailleurs, bien que les patients sous hydroxyurée connaissent moins d'hospitalisation que ceux qui ne le prennent pas [20], son introduction en 1998 aux États-Unis n'a pas entraîné une diminution significative du niveau d'hospitalisation, comme le montre une étude menée entre 1998 et 2008 [29].

La non-observance ainsi que la constance dans le temps des motifs d'hospitalisation interrogent sur la représentation que se fait le malade tant de sa maladie que de ses conséquences. Ainsi, se pose le problème de l'impact de cette représentation sur son comportement vis-à-vis du traitement : la représentation de la maladie que se fait le patient pourrait en effet expliquer des comportements de non-observance.

\subsection{La représentation de la maladie}

Le modèle développé en 1980 par Howard Leventhal, Daniel Meyer et David R. Nerenz, connu comme le Modèle de Sens commun d'Auto-régulation (Common sens Modele of Self Regulation, CSM), apparaît comme la synthèse de deux grands courants de théorisation du comportement en psychologie de la santé : les théories comportementales et les théories sociocognitives et émotionnelles [22].

Suivant le modèle de Leventhal, la représentation de la maladie stockée en mémoire peut être définie comme le fruit de la synthèse des connaissances propres du malade sur sa maladie, des informations sur sa maladie apportées par des sources extérieures reconnues et de l'expérience personnelle de la maladie et de ses symptômes.

\subsubsection{Les trois postulats fondant le modèle de Sens Commun (CSM)}

1. L'individu est pensé comme un agent actif résolvant des problèmes liés à sa santé. En cherchant à comprendre son état de santé, il tentera de réduire l'écart entre la perception qu'il en a 
et celui auquel il aspire.

2. Dans le domaine de la santé, les représentations profanes des patients s'opposent aux représentations scientifiques véhiculées par le corps médical. Elles sont personnelles et se construisent à partir du vécu propre de la maladie, du discours émanant de tiers de confiance et du discours médical.

3. La représentation de la maladie ainsi construite conduit à l'adaptation à cette dernière puis l'individu va procéder aux évaluations subséquentes qui confèrent le caractère dynamique au modèle.

\subsubsection{Les cinq dimensions de la représentation de la maladie}

Le modèle d'Auto-régulation de Sens commun postule une réponse parallèle tant comportementale que cognitive à la représentation construite par le malade de sa maladie dont cinq dimensions peuvent être évaluées $[4,40]$ et qui entretiennent un lien avec l'observance ou l'adhésion thérapeutique comme le montre une revue de littérature de 2012 de 11 études [18] portant sur l'asthme, le diabète, l'infarctus du myocarde, le glaucome, la douleur chronique et la tuberculose :

1. identité : l'étiquette ou nom qui est donné à la maladie et aux symptômes qui y sont associés. Elle détermine la sévérité perçue de sa maladie. Dans l'hypertension, l'identité perçue de la maladie est liée significativement et positivement avec l'observance thérapeutique, alors que cette relation n'existe pas dans l'asthme, par exemple ;

2. cause: l'ensemble des idées personnelles sur les causes ayant entraîné sa maladie, même si elles ne sont pas scientifiquement correctes. Compte tenu de l'hétérogénéité des causes avancées pour expliquer la maladie, il est difficile de généraliser des résultats pour l'observance ou l'adhésion thérapeutique ;

3. chronologie/cyclicité: les croyances prédictives sur la durée de sa maladie. Elles auraient une influence sur l'observance par la perception de la nécessité de prendre son traitement. Par ailleurs, les patients qui perçoivent leur maladie comme variable au cours du temps sont moins enclins à prendre leur traitement comme prescrit, dans l'hypertension par exemple ;

4. conséquences : les représentations personnelles sur les conséquences de la maladie et leurs impacts sur la vie du patient n'entraînent pas nécessairement une augmentation de 
l'observance thérapeutique. Pour l'asthme, les patients avec une forte perception présentent une mauvaise observance, alors que dans l'hypertension, la réaction est inverse. Néanmoins, selon les auteurs, les patients suivant leur prescription sont en meilleure santé et perçoivent moins les effets négatifs de la maladie sur leur vie, ce qui fait de cette dimension un bon indicateur d'observance ;

5. curabilité/contrôlabilité : croyances quant à la guérison ou au contrôle possible sur la maladie et l'implication du patient dans ce processus. La croyance dans la capacité du traitement à réduire les symptômes (curabilité) est liée à une meilleure observance thérapeutique, alors que la perception de son contrôle personnel sur la maladie est lié à une faible observance. Les patients (e.g. hypertension) ayant un fort sentiment de contrôle investissent d'autres méthodes pour contrôler leur maladie (e.g. Sport, nutrition, etc.).

La variation des résultats en fonction de la maladie chronique nous incite à étudier plus spécifiquement les liens existants entre la représentation de la drépanocytose et l'observance qui n'ont jamais été spécifiés. Or :

- - la drépanocytose est une maladie chronique d'origine génétique se déclarant d'emblée, et non pas acquise, ce qui la rend spécifique par rapport aux autres pathologies étudiées ;

- $\quad$ - nous ne disposons pas de connaissance sur la façon dont les personnes atteintes se représentent leur maladie. En particulier, la notion de cause de la maladie paraît utile à explorer. Des études anthropologiques [19] soulignent la superposition possible avec des croyances magico-religieuses de la causalité génétique connue.

\subsection{Objectifs et hypothèse de la recherche}

Cette étude a pour objectif d'étudier la représentation de la drépanocytose chez les malades adultes à partir des neuf dimensions de l'IPQ-R et de définir un lien entre la représentation que se font les patients de leur drépanocytose et leur observance thérapeutique.

L'objectif est de mieux connaître les profils des patients afin d'améliorer leur prise en charge; enfin, l'importance au niveau clinique de cette étude est de mettre en place une information. 


\subsubsection{Hypothèse de recherche}

Étant donné que les études sur les maladies chroniques ont montré un lien entre la représentation de la maladie et l'observance thérapeutique, nous nous attendons à retrouver ce lien chez les personnes atteintes de drépanocytose.

\section{Méthodologie}

\subsection{Lieux de recueil de données}

Le recueil de donnée a été effectué dans deux CHU de la région parisienne (Hôpital Européen George Pompidou [HEGP], en consultation et en hospitalisation; Hôpital Henri Mondor, en consultation).

\subsection{Participants à l'étude}

L'échantillon de cette étude est composé de 79 patients adultes homozygotes volontaires suivis en consultation. Seuls trois patients ont refusé de répondre au questionnaire. L'échantillon est composé de $48,1 \%$ d'homme $(n=38)$ et $51,9 \%$ de femme $(n=41)$. La moyenne d'âge est de 34,42 ans $(\sigma=11,108 ;[16 ; 70])$. Dans notre échantillon, les hommes sont plus jeunes que les femmes et, les patients de l'HEGP sont plus jeunes que les patients d'Henri Mondor ( $t$ varie entre $6,409$ et $-3,449 ; p<0,05)$. Les réponses aux différents questionnaires (IPQ-R, BMQ et MMAS) ne sont pas influencées par le lieu de recueil de données ou par le sexe des répondants $(t$ varie entre $-0,927$ et 1,$723 ; p>0,05)$.

\subsection{Outils psychométriques et socio-démographiques}

\subsubsection{Données sociodémographiques et médicales}

La première partie du questionnaire explore les données sociales (âge, lieu de naissance, niveau socio-culturel), familiales (célibat, parentalité), scolaires et médicales (lieux de diagnostic 
et de premier traitement, nombre d'hospitalisation) du répondant. Une évaluation du bien-être en 10 points a été élaborée pour l'étude.

\subsubsection{Mesure de l'observance thérapeutique}

Échelle auto-rapportée d'observance construite par Morisky et al. (25) : Morisky Medication Adherence Scale (MMAS) (validation française de 2012 par Korb-Savoldelli et al. (17)). Elle est composée de 8 items, 7 à réponse dichotomique oui/non, le dernier par une échelle de temps en 5 points. Un score inférieur à 6 indique une mauvaise observance, entre 6 et $<8$, une observance moyenne et un score de 8 une bonne observance.

\subsubsection{Mesure des croyances sur les traitements}

Questionnaire de Croyances sur les Traitements (Beliefs about Medecines Questionnaire $(B M Q))$ Horne et al en 1997, validation française Fall et al. $(6,14)$. Vise à évaluer les croyances des patients sur leur traitement. Cette échelle est composée de 18 items cotées sur une échelle de Lickert en cinq points de 1 à 5 et répartis en trois dimensions :

1. la nécessité (necessity scale) des traitements recommandés pour contrôler la maladie,

2. leurs préoccupations (concerns scale) à prendre les traitements recommandés,

3. les croyances générales sur les médicaments, incluant deux sous-échelles concernant la perception de la nuisance possible des traitements (harm scale) et de l'abus de prescription des médecins (overuse scale).

Un résultat composite peut être retiré de ces dimensions: la différence NécessitéPréoccupations. La nécessité et les préoccupations évaluent les attitudes positives et négatives vis-à-vis du traitement. Une indication de l'importance relative de ces attitudes pour le patient peut être estimée en faisant la différence entre le score de nécessité et celui de préoccupations. La différence Nécessité-Préoccupations a une amplitude de -20 à 20. Un score négatif souligne l'importance des attitudes négatives et, inversement, un score positif indique une prépondérance des attitudes positives vis-à-vis du traitement. Selon les concepteurs du questionnaire, cette différence est une mesure indirecte de l'adhésion thérapeutique. 


\subsubsection{Mesure de la représentation de la maladie}

Questionnaire de Perception de la Maladie (Illness Perception Questionaire Revised $(I P Q-R))$ : issu de la révision de l'IPQ (développé par Weinman et al. en 1996 [20]) par MossMorris et al. en 2002 [27]. Comme le proposent les auteurs de cette révision, nous avons adapté ce questionnaire au cas particulier de la drépanocytose. Deux modifications ont été apportées, d'abord sur la spécification de la maladie, en remplaçant le terme générique «maladie » par « drépanocytose » et en adaptant l'échelle de causalité.

Aux cinq dimensions initiales, l'IPQ-R couvre désormais neuf dimensions cotées sur une échelle de Lickert en cinq points de 1 à 5, sauf la dimension Identité qui est dichotomique oui/non :

1. Identité : représente l'importance de la maladie pour le patient. Plus il considère de symptôme comme étant associé à sa drépanocytose, plus celle-ci est perçue comme sévère ou envahissante.

Une liste de 14 symptômes (douleurs, gorge irritée, nausées, difficultés respiratoires, perte de poids, fatigue, articulations raides, yeux irrités, respiration sifflante, maux de tête, lourdeur d'estomac, difficultés pour dormir, vertiges, perte de force) est proposée aux répondants dans laquelle deux sont directement associés à la drépanocytose : les douleurs et les difficultés respiratoires.

2. Durée de la maladie : la temporalité de la maladie est évaluée selon son caractère aiguë ou long/chronique. La Durée est évaluée par 6 items ( $\mu^{\text {théo }}: 18$; $[\min : 6$; $\left.\max : 30]\right)$. Un score élevé indique une perception de la chronicité de la maladie.

3. Conséquences négatives perçues : permet de mettre en évidence l'impact de la maladie sur la vie du malade et ses répercussions dans son quotidien. Conséquences négatives perçues est évalué par 6 items $\left(\mu^{\text {théo }}: 18 ;[\min : 6 ; \max : 30]\right)$. Plus le score est important, plus les conséquences négatives sont perçues.

4. Contrôle personnel : évalue la perception des actions que le malade pourrait mettre en place pour limiter ou arrêter les effets négatifs de la maladie (e.g. douleur). Le Contrôle personnel est évalué par 6 items ( $\left.\mu^{\text {théo }}: 18 ;[\min : 6 ; \max : 30]\right)$.

5. Efficacité perçue des traitements : évalue la croyance en la capacité des médicaments à 
être efficace dans le traitement de la maladie. L'efficacité des traitements est évaluée par 5 items $\left(\mu^{\text {théo }}: 15 ;[\min : 5 ; \max : 25]\right)$.

6. Cohérence de la perception de la maladie: montre la compréhension que les patients ont de leur propre maladie. La dimension de Cohérence est évalué par 5 items ( $\mu^{\text {théo }}: 15$; [min : $5 ; \max : 25])$.

7. Cyclicité des symptômes : explore la variation du ressenti des symptômes. La Cyclicité est appréciée par 4 items $\left(\mu^{\text {théo }}: 12 ;\right.$ [min : $\left.4 ; \max : 20\right]$ ). Un score élevé indique une perception de la cyclicité des symptômes.

8. Représentations émotionnelles : met en évidence les émotions négatives engendrées par la maladie. Plus le score est élevé, plus les émotions négatives prédominent.

9. Échelle des causes de la maladie : explore différentes causes perçues possibles pour expliquer l'apparition de la maladie.

Nous avons adapté la sous-échelle « cause de la maladie » en ajoutant des items selon les apports de Stéphanie Gernet $[9,10]$ :

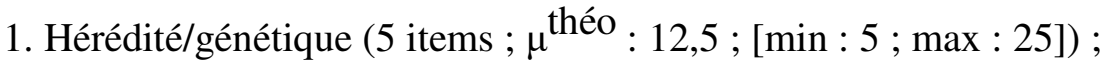

2. Origine géographique, Environnement et le climat $\left(4\right.$ items $; \mu^{\text {théo }}: 12 ;[\min : 4$; $\max : 20])$;

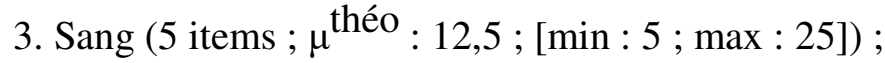

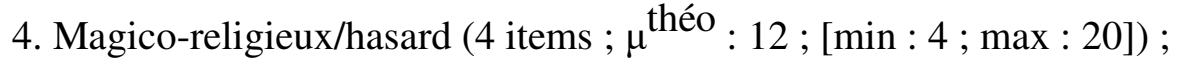

Nous avons conservé les deux facteurs complémentaires liés aux comportements composés des items présents initialement dans la sous-échelle «Causes de la maladie » de l'IPQ-R :

1. Comportement actif (ensemble des actions ou des comportements réalisé par le patient qui aurait pu déclencher la maladie) $\left(7\right.$ items $\left.; \mu^{\text {théo }}: 21 ;[\min : 7 ; \max : 35]\right)$;

2. Comportement passif (ensemble des situations extérieures au patient qui auraient pu être à l'origine de la maladie) $\left(7\right.$ items ; $\left.\mu^{\text {théo }}: 21 ;[\min : 7 ; \max : 35]\right)$.

L'échelle des causalités est ainsi composée de 32 items mélangés, cotés par une échelle de Lickert de 1 à 5. L'ensemble des items doit être coté par le répondant, donc plusieurs causalités peuvent être retenues par un seul malade comme source de sa drépanocytose. 
Cette échelle de cause pour la drépanocytose présente une bonne consistance inter-items, le coefficient $\alpha$ de Cronbach pour l'ensemble de l'échelle des causes de la maladie est de 0,87. De même, elle présente une bonne consistance inter-échelle $(\alpha=0,78)$.

Afin d'identifier quelles attributions causales étaient signifiantes pour chaque répondant et combien il en acceptait, nous avons suivi la procédure adoptée par Horne et Weinman [15] consistant, pour chaque répondant, à faire la moyenne de chaque dimension et de la recoder selon l'échelle de Lickert en fonction de l'acceptation du patient à la causalité (de d'accord à tout à fait d'accord $=1$; de pas du tout d'accord à indifférent $=0$ ).

\section{Procédures}

\subsection{Comité de protection des personnes et Comité d'éthique}

La présente étude a reçu les validations éthiques suivantes :

- $\quad \square \quad$ Comité de Protection des Personnes Île-de-France Ambroise Paré (8) $\mathrm{n}^{\circ}$ obs.16.03 du 04 avril 2016 ;

- $\quad \square \quad$ Comité d'Éthique de Necker-Enfants Malades (CENEM) n²016-DB-13 du 27juin 2016 ;

\subsection{Procédure de recueil de données}

Les participants sont rencontrés individuellement par l'investigateur après avoir été orientés par leur médecin et avoir rempli un consentement éclairé. La complétion du questionnaire se fait à l'hôpital, en individuel, dans un bureau mis à la disposition de l'investigateur. La passation durait entre 20 et $40 \mathrm{~min}$.

\subsection{Statistiques}

La normalité de distribution des variables introduites dans cette étude a été vérifiée par un test de Kolmogorov-Smirnov avec la correction de signification de Lilliefors. Une analyse descriptive a été menée pour résumer ces données (moyenne, écart-type, chi² ${ }^{2}$ et comparaison des 
moyennes par le $t$ de student ou le calcul du coefficient $F$ de Fisher). Pour démontrer notre hypothèse, nous avons utilisé un test de corrélation au moyen du calcul du coefficient $r$ de corrélation de Pearson et des ANOVA. Une modélisation a été effectuée par régression. Tous les résultats ont été traités à l'aide du logiciel SPSS 20 pour Microsoft.

\section{Résultats}

\subsection{Présentation des données sociodémographiques et des résultats aux auto-questionnaires}

\subsubsection{Analyse des données sociodémographiques}

Insertion tableau I : Synthèse descriptive des données socio-démographiques.

Notre population est composée de façon équilibrée de femmes et d'hommes $(51,9 \%$; 48,1\%). Les participants sont nés pour moitié en France (31,6 \% en métropole et 12,7\% dans les $\mathrm{DOM} / \mathrm{TOM}$ ) et pour moitié en Afrique $(51,9 \%)$. Ils ont un niveau d'étude médian au baccalauréat. Presque $60 \%$ vivent seuls (séparés, divorcés...). Ils ont en moyenne 1,05 enfants ( $\sigma$ $=1,23$ ), avec une différence selon le lieu de naissance (Afrique $>$ DOM-TOM $>$ France $-F=$ $5,11 ; p=0,003)$.

La drépanocytose a été diagnostiquée à $59,5 \%$ en France $(50,6 \%$ en métropole et 8,9\% dans les DOM/TOM) et 35,4\% en Afrique et la majorité $(81 \% ; \mathrm{n}=64)$ des patients présentent un phénotype SS, $12,7 \%(\mathrm{n}=10)$ sont $\mathrm{SC}$, et $6,4 \%(\mathrm{n}=5)$ présentent une drépanocytose $\beta$ thalassémique.

Les patients rapportent en moyenne 3,33 hospitalisations $(\sigma=3,90)$ par an au cours des cinq dernières années ([min : 0 ; max : 20]). Un quart des patients rapportent plus de quatre hospitalisations par an. Le profil des participants à l'étude est concordant avec celui retrouvé dans d'autres études [38].

Les participants recrutés à Henri Mondor présentent un profil sociodémographique significativement différent de HEGP : ils sont plus âgés, viennent majoritairement d'Afrique, sont plus souvent en couple et rapportent moins d'hospitalisations. Nous n'avons pas d'éléments pour expliquer ces différences sociodémographiques entre les deux populations. Cependant, les 
résultats aux auto-questionnaires ne sont pas influencés par le lieu de recueil de données ou le sexe.

Insertion tableau II : Résultats aux auto-questionnaires.

5.1.2. Présentation des résultats des déterminants de l'adhésion (BMQ) et de l'observance thérapeutique (MMAS)

Insertion tableau III : Corrélation des dimensions de la représentation (IPQ-R) avec les dimensions de l'adhésion (BMQ) et de l'observance (MMAS) thérapeutique.

\subsubsection{Observance thérapeutique (Morisky Medication Adherence Scale)}

La moyenne de notre population à l'échelle d'observance MMAS est de $\mathrm{m}=4,04$ ( $\sigma$ $=1,81 ;[\min : 0 ; \max : 7])$ indiquant en moyenne une mauvaise observance de notre population. Zéro pour cent de notre population a un score de 8 correspondant à une observance parfaite, alors que $77,4 \%$ ont un score compris entre 6 et 8 correspondant à une faible observance et que $22,6 \%$ présentent une observance moyenne.

\subsubsection{Adhésion thérapeutique (Beliefs about Medecine Questionnaire).}

La différence Nécessité-Préoccupations a un score moyen de 3,77 ( $\sigma=5,89$; [ $\min :-15$; $\max : 15]), 25,7 \%$ de notre population ont un score compris entre 0 et -15 , et $16,5 \%$ ont un score supérieur à 10. Ce score indique une prédominance de la nécessité perçue d'appliquer correctement son traitement (attitude positive) sur les préoccupations liées aux effets secondaires possibles (attitude négative). Cependant, 25,7 \%, soit un quart des malades, ont un score négatif, indiquant une attitude négative vis-à-vis des traitements. Cette différence peut être utilisée comme indicateur indirecte de l'observance thérapeutique puisque la différence NécessitéPréoccupations est corrélée positivement $(r=0,45 ; p<0,01)$ avec le score MMAS.

\subsubsection{La représentation de la drépanocytose (Illness Perception Questionnaire-Revised)}

\subsubsection{Identité perçue}


Les patients associent en moyenne $5,83(\sigma=2,64)$ symptômes $\left(\mu^{\text {théo }}: 6,5 ;[\min : 0\right.$; $\max : 13]$ ) à la drépanocytose (médiane de 6). Près de $40 \%$ des patients en rapportent plus de six dont $62,5 \%$ plus de sept symptômes. Les plus fréquemment rapportés sont : la douleur $(92,4 \%)$, la fatigue $(73,4 \%)$, les difficultés respiratoires $(64,6 \%)$, la perte de force $(63,3 \%)$ et les articulations raides $(55,7 \%)$.

\subsubsection{Perception de la chronologie : durée et cyclicité}

Dans notre population, la perception de la durée de la maladie est de $\mathrm{m}=23,71(\sigma=4,92$; [min : $10 ; \max : 30])$ et la Cyclicité est en moyenne de $\mathrm{m}=14,17(\sigma=2,97 ;[\min : 7$; $\max$ : 20]). Ces résultats sont supérieurs à la moyenne théorique de ces dimensions. Plus de la moitié de la population a un score de durée supérieur à 24,5 et un score de Cyclicité supérieur à 15.

Bien que la drépanocytose soit perçue par $60 \%(\mathrm{n}=42)$ des patients comme une maladie chronique qui va durer dans le temps, $40 \%(\mathrm{n}=28)$ ne perçoivent pas cette chronicité alors que cette maladie est chronique, incurable, et qu'ils en sont atteints depuis leur naissance ; 48,7\% (n =38) ne perçoivent pas la variation dans le temps des manifestations symptomatiques alors même que les manifestations douloureuses réapparaissent périodiquement et sont imprévisibles.

\subsubsection{Perception des conséquences négatives de la drépanocytose}

La moyenne obtenue $\mathrm{m}=20,81(\sigma=4,76 ;[\min : 8 ; \max : 29])$ est supérieure à la moyenne théorique. Cinquante pour cent de la population ont un score de Conséquence supérieur à $21 ; 25 \%$ un score supérieur à 25 ; en revanche, 33,8\% ont un score inférieur à 20 . Ce résultat montre que la moitié des personnes malades ressentent un impact négatif de la drépanocytose sur leur vie et un quart perçoivent leur vie comme très impactée par leur maladie. Cependant, un tiers ne perçoivent pas de conséquences négatives de la drépanocytose sur leur existence.

\subsubsection{Compréhension rapportée de sa maladie}

La moyenne obtenue est de 18,66 ( $\sigma=3,40$; [ $\min : 8$; $\max : 25])$ supérieure à la moyenne théorique. La distribution des centiles nous indique que plus de $54 \%(\mathrm{n}=41)$ de la population a un score de Cohérence supérieur à cette moyenne. Ce résultat signifie que près de $46 \%(\mathrm{n}=35)$ de la population d'étude n'ont pas une compréhension claire de sa maladie, c'est-à- 
dire qu'elle n'arrive pas à donner un sens personnel aux symptômes ou à la maladie.

\subsubsection{Perception de la curabilité et de la contrôlabilité de la drépanocytose}

La perception de la contrôlabilité de la maladie est en moyenne de $\mathrm{m}=21,84(\sigma=4,10$; [min : 12 ; $\max : 30])$ et l'efficacité des traitements de $\mathrm{m}=17,67(\sigma=3,44$; $[\min : 5 ; \max : 24])$ supérieures à leurs moyennes théoriques.

La distribution des résultats montre que $62,5 \%$ de la population ont un score de perception de l'efficacité des traitements supérieur à cette moyenne. Tandis que pour la contrôlabilité, près de 50 \% de la population ont un score supérieur à sa moyenne.

Dans leur majorité, les participants perçoivent leur traitement comme efficace pour lutter contre les symptômes de la drépanocytose; cependant, plus d'un tiers ne perçoivent pas le traitement comme efficace pour lutter contre la maladie et la moitié seulement ont confiance en leur capacité de mettre en place des actions pour limiter ou arrêter les effets négatifs de la maladie.

\subsubsection{Relation entre les différentes représentations de la maladie}

Les personnes ne percevant pas les conséquences négatives présentent des différences significatives avec le reste de la population: un score moyen de bien-être rapporté et une perception de leur capacité à contrôler la drépanocytose grâce au traitement (contrôle traitement) supérieur $(t=-3,00 ; p=, 004$ et $t=-2,34 ; p=, 023)$ aux autres patients. Ils associent moins de symptômes à la drépanocytose que les autres patients $(t=3,23 ; p=, 002)$ et ont une préoccupation liée aux possibles effets secondaires du traitement plus faible $(t=2,89 ; p$ $=, 006)$; en revanche, ils ont une moins bonne perception de la chronicité et de la cyclicité de la maladie $(t=2,21 ; p=, 033$ et $t=2,42 ; p=, 021)$ et, paradoxalement, perçoivent moins la nécessité de prendre son traitement ( $t=2,15 ; p=, 037)$.

\subsubsection{Causes acceptées de sa drépanocytose}

Insertion tableau IV : Taux d'acceptation des causes (IPQ-R).

La première causalité adoptée montre que la drépanocytose est considérée comme une 
maladie dont la cause est liée au sang $(60,6 \%)$ et à l'hérédité $(56 \%)$ pour plus de la moitié des patients. L'attribution « sang » est le fruit de la confusion entre la cause de la maladie (une mutation génétique) et l'effet de cette maladie (une malformation de l'hémoglobine). Elle reste proche des causes réelles ayant généré la maladie, sans se superposer cependant. Ainsi, un lien, modéré et positif, significatif $(r=0,48 ; p<0,001)$ existe entre la cause Hérédité/génétique et la cause Sang : les patients retenant la causalité Hérédité/Génétique acceptent également à 74,40\% la causalité Sang et ceux retenant la causalité Sang acceptent aussi à 70,70\% la causalité Hérédité/Génétique.

Les autres causes sont plus rarement évoquées. L'origine géographique (12,3\%) renvoie la drépanocytose à une maladie de l'Afrique. Une minorité attribue la maladie à une cause comportementale $(6,5 \%)$ : par exemple fumer ou boire de l'alcool ; et un seul attribue la maladie à une cause magico-religieuse $(1,3 \%) ; 10,3 \%$ de notre population excluent les causalités connues (Hérédité ou Sang) : 2,53\% $(\mathrm{n}=2)$ rejettent à la fois Hérédité et Sang, 5,06\% $(\mathrm{n}=4)$ acceptent comportement passif, $1,3 \%(\mathrm{n}=1)$ acceptent comportement actif et 1,3\% $(\mathrm{n}=1)$ Magie.

Les patients acceptant soit la cause Hérédité/génétique soit la cause Sang comme origine de la drépanocytose rejettent entre $85,4 \%$ et $97,6 \%$ toute autre causalité pour leur maladie. Il apparait donc que seuls $56 \%$ des participants sont en mesure d'identifier avec justesse la cause, génétique de leur maladie, maladie dont ils sont pourtant porteurs depuis la naissance et qui les conduit à consulter régulièrement les médecins en consultation spécialisée ou en hospitalisation.

\subsection{Prédicteurs de l'observance thérapeutique}

Insertion tableau $V$ : Prédicteurs de l'influence de la représentation de la maladie sur l'observance thérapeutique et sur l'adhésion thérapeutique - régression linéaire.

Nous avons effectué une modélisation du lien entre le score d'observance thérapeutique et les variables sociodémographiques, les dimensions de la représentation de la maladie (IPQ-R) et l'indicateur d'adhésion thérapeutique $(\mathrm{N}-\mathrm{P})$ à partir de régressions linéaires.

Trois dimensions de la représentation de la maladie de l'IPQ-R prédisent significativement l'observance thérapeutique : l'efficacité perçue des traitements $(\beta=, 34)$, la durée perçue de la 
maladie $(\beta=, 31)$ et la représentation émotionnelle $(\beta=-, 32)$. Ainsi, plus les patients perçoivent l'efficacité des traitements et leur maladie comme chronique, moins ils ont d'émotions négatives liées à la drépanocytose et plus ils sont observants. Ces trois dimensions expliquent 29,2\% de la variance totale de l'observance $\left(\mathrm{R}^{2}\right)$.

Les mêmes dimensions de la représentation de la maladie de l'IPQ-R prédisent également l'adhésion thérapeutique: l'efficacité perçue des traitements $(\beta=, 37)$, la durée perçue de la maladie $(\beta=, 36)$ et la représentation émotionnelle $(\beta=-, 36)$. Ainsi, plus les patients perçoivent l'efficacité des traitements et leur maladie comme chronique, moins ils ont d'émotions négatives liées à la drépanocytose et plus ils sont adhérents. Ensemble, ces trois variables expliquent $33,8 \%\left(\mathrm{R}^{2}\right)$ de la variance totale de l'adhésion thérapeutique.

La différence Nécessité-Préoccupations du BMQ, considérée par certains auteurs comme une mesure de l'adhésion thérapeutique, s'avère ici davantage une mesure alternative à l'observance évaluée par la MMAS $(\mathrm{r}=0,44 ; p=0,00)$ : d'une part, les résultats sont très similaires sur les deux mesures, d'autre part, la variable N-P n'apparait pas comme prédicteur de l'observance. Aucune variable sociodémographique ou médicale n'apparaît comme prédicteur de l'observance dans notre population.

\section{Discussion}

Ces résultats suggèrent une influence de la représentation de la maladie sur l'observance thérapeutique, même dans le cas d'une maladie génétique à l'étiologie connue dont les symptômes se manifestent dès le début de la vie.

\subsection{Première implication : sur les connaissances et les représentations des personnes atteintes de drépanocytose de leur maladie}

Notre population d'étude est relativement jeune (environ 35 ans) pour laquelle un peu moins de la moitié rapportent un fort impact de la maladie et un-quart un très fort impact. Plus de la moitié éprouvent les conséquences négatives sur leur vie et, pour un-quart, ces conséquences semblent être extrêmes. La drépanocytose apparaît bien comme une maladie dont les répercussions sur la vie des patients est sévère. 
Dans le même temps, près de la moitié des patients ne reconnaîtraient pas l'étiologie génétique de la drépanocytose et auraient une méconnaissance globale de la maladie : ils ne semblent pas reconnaître la drépanocytose comme une maladie chronique ni percevoir la variation temporelle des symptômes. Ceci a des conséquences sur la perception qu'ils ont du traitement. Ainsi, près de la moitié des patients ne perçoivent pas leur traitement comme pouvant les aider à contrôler leur maladie : près de la moitié d'entre eux ne reconnaissent pas la nécessité de leur traitement et plus de la majorité rapportent des préoccupations à prendre leur traitement.

La moitié des patients ne se sentent pas capables de réguler leurs symptômes par leurs propres actions. Plus encore, plus de la majorité des personnes considèrent le traitement comme une nuisance avec plus d'un quart ayant des croyances franchement négatives sur leur traitement et près de la moitié pense que les médecins abusent des médicaments.

\subsection{Seconde implication : qui sont les patients les plus observants ?}

La représentation émotionnelle, l'efficacité perçue des traitements et la durée perçue de la maladie influencent l'observance thérapeutique pour $29,2 \%$, suggérant que la représentation que se font les personnes atteintes de drépanocytose aurait une influence sur leur comportement envers le traitement. On constate que les patients les plus observant perçoivent plus les conséquences négatives de la drépanocytose, notamment la cyclicité des symptômes, ce qui se traduit par une perception plus forte de l'intérêt de la prise des traitements. Ils montrent une prédominance de la nécessité perçue d'appliquer correctement leur traitement (attitude positive) sur les préoccupations liées aux effets secondaires possibles (attitude négative), sur la nuisance des traitements et sur l'abus de prescriptions médicales. De plus, les émotions négatives envers la maladie semblent jouer un rôle limitatif d'une bonne observance thérapeutique.

Nos résultats sont congruents avec ceux relevés par Kucukarslan en 2012 [18] sur le lien

entre la représentation de la maladie (selon le modèle de Leventhal) et l'observance dans sept pathologies non génétiques (asthme, glaucome, tuberculose, diabète, hypertension, maladies cardio-vasculaires, douleurs chroniques) : l'efficacité perçue des traitements et la durée perçue de la maladie ont une influence positive sur l'observance thérapeutique. En revanche, le rôle négatif des représentations émotionnelles sur l'observance thérapeutique n'est pas apparu, comme c'est le cas dans la présente étude sur la drépanocytose. 
Dans notre modèle, les variables sociodémographiques (âge, sexe, lieu de naissance, niveau d'étude, etc.) ou médicales (phénotype, nombre d'hospitalisations, etc.) n'interviennent pas, laissant supposer que l'observance thérapeutique, dans le cas de la drépanocytose, ne serait pas sensible au type de drépanocytose, à sa sévérité médicale ou à des variables ethnologiques et sociales. Nous n'avons pas d'explication satisfaisante autre que la faiblesse de notre échantillon : on se serait attendu à ce que le niveau d'étude, le statut socio-économique ou le nombre d'hospitalisation rapporté aient une influence sur l'observance thérapeutique, comme le montre la méta-analyse de 569 études réalisée par DiMatteo en 2004 [5].

\section{Conclusion}

L'étude nous permet de confirmer l'hypothèse selon laquelle les représentations de la maladie varient également dans une maladie génétique dont l'étiologie est clairement identifiée par le corps médical. Cette représentation aurait une influence sur l'observance thérapeutique des patients atteints par une maladie génétique. Ces résultats nous indiquent également que le modèle d'autorégulation de Leventhal permet de comprendre les comportements d'ajustement à la maladie chronique, et ici spécifiquement à la drépanocytose. Comme le postule le modèle, nous voyons que l'individu construirait une représentation de sa maladie qui va guider l'adaptation à cette dernière.

\subsection{Limites et points forts de l'étude}

Le travail présenté donne une vision globale des déterminants de l'observance dans une population adulte de personnes atteintes par la drépanocytose mais pèche par la faiblesse de son échantillon. En particulier, l'absence d'influence des données médicales (e.g. nombre d'hospitalisation rapportée) ou des données sociales (e.g. niveau d'étude) est étonnante et demande à être confirmée.

Cependant, cette première recherche a permis de mettre en évidence un premier modèle pouvant expliquer la variation de l'observance thérapeutique. Il implique l'efficacité perçue des traitements, la chronicité perçue de la maladie et les représentations émotionnelles. L'indicateur d'adhésion thérapeutique proposé par Horne est également apparu ici comme une mesure 
alternative de l'observance plus que comme une mesure complémentaire de l'adhésion. De nouvelles études devraient permettre d'obtenir un éclairage plus précis sur cette question.

\subsection{Pistes pour de futures recherches}

Le modèle proposé explique 29,2 \% de la variance de l'observance et 33,8 \% de l'adhésion thérapeutique : les deux tiers de ses dimensions restent à expliquer. Un échantillon plus important permettra d'étoffer ce premier modèle. Pour compléter notre compréhension de l'observance thérapeutique deux pistes sont à explorer :

a) la littératie en santé : les caractéristiques de la population étudiée invitent à essayer de mieux comprendre pourquoi les patients ne connaissent pas mieux leur maladie avant d'organiser un travail d'information et d'éducation thérapeutique plus systématique. La littératie en santé est un concept relativement nouveau dans le domaine de la psychologie de la santé qui peut se définir comme «les habilités sociales et cognitives qui déterminent la motivation et la capacité d'un individu à avoir accès, comprendre et utiliser des information qui vont promouvoir et maintenir une bonne santé » [30];

b) les émotions et la douleur: la représentation émotionnelle liée à la maladie est superficiellement interrogée par l'IPQ-R (6 items) et uniquement d'un point de vue négatif (e.g. «Je déprime quand je pense à la drépanocytose », « Ma drépanocytose me fait peur »). Pour avoir une meilleure perception des représentations émotionnelles, il faut étendre la mesure à leur valence, leur traitement, et la capacité à les ressentir ou les exprimer. De même pour le vécu douloureux qui chevauche les représentations émotionnelles.

La drépanocytose se caractérisant par des crises de douleur imprévisibles, soudaines et d'une intensité extrême, il faut en effet caractériser ce vécu douloureux, en particulier par la compréhension de la dramatisation associée à ces crises et aux croyances associées.

Conflit d'intérêt : les auteurs déclarent ne pas avoir de lien d'intérêt.

\section{Références}

[1] Anie KA. Psychological complications in sickle cell disease. Br J Haematol 2005;129:723 $\square 9$.

[2] Bachir D. La Drépanocytose. Rev Fr Lab 2000;(324):29 $\square 35$. 
[3] Barofsky I. Compliance adherence and the therapeutic alliance: step in the development of self-care. Soc Sci Med 1978;12:369 $\square 76$.

[4] Cameron L, Moss-Morris R. Illness-relatied cognition and behaviours. In: French D, Vedhara K, Kaplein AA, Weinman J, éditeurs. Health Psychology 2e. Londres: Blackwell; 2010. P. $149 \square 61$.

[5] DiMatteo MR. Variations in Patients' Adherence to Medical Recommendations: A Quantitative Review of 50 Years of Research. Med Care 2004;42:200 $\square 9$.

[6] Fall E, Gauchet A, Izaute M, Horne R, Chakroun N. Validation of the French version of the Beliefs about Medicines Questionnaire (BMQ) among diabetes and HIV patients. Rev Eur Psychol AppliquéeEuropean Rev Appl Psychol 2014;64:335 43 .

[7] Galactéros F. Numéro thématique - La drépanocytose en France: des données épidémiologiques pour améliorer la prise en charge. Institut Nationale de Veille Sanitaire, éditeur. Bull épidémiologique hebdomadaire 7 mars 2012;(27 $\square 28): 311 \square 29$.

[8] Gardner K, Douiri A, Drasar E, Allman M, Mwirigi A, Awogbade M, et al. Survival in adults with sickle cell disease in a high-income setting. Blood 2016;128:1436 $\square$.

[9] Gernet S. Perception et représentation de la drépanocytose [Internet]. 2010 [cité 14 oct 2015]. Disponible sur: http://u-bordeaux2medtrop.org/doc/Soutenances/These/Gastro_enterites_aigues_infectieuses_Mayotte_2010_Bouge r.pdf

[10] Gernet S, Mestre C, Runel-Belliard C. Représentations émotionnelles de la maladie chez 22 enfants drépanocytaires. Neuropsychiatr Enfance Adolesc 2011;59:404 $\square 10$.

[11] Godeau B, Noël V, Habibi A, Schaeffer A, Bachir D, Galacteros F. La drépanocytose chez l'adulte: quelles urgences pour l'interniste? Rev Médecine Interne 2001;22:440-51.

[12] Gomes E, Castetbon K, Goulet V. Mortalité liée à la drépanocytose en France : âge de décès et causes associées (1979-2010). Bull épidémiologique hebdomadaire 2015;8:142 $\square 50$.

[13] Haynes RB, éditeur. Compliance in health care. Baltimore, Md.: Johns Hopkins Univ Press; 1979.

[14] Horne R, Weinman J, Hankins M. The Beliefs about Medecines Questionnaire: the development and evaluation of a new method for assessing the cognitive representation of medication. Psychol Health 1997;14:1 $\square 24$.

[15] Horne R, Weinman J. Self-regulation and selfmanagement in asthma: exploring the role of 
illness perceptions and treatment beliefs in explaining nonadherence to preventer medication. Psychol Health 2002;17:17 $\square 32$.

[16] INSERM. Drépanocytose [Internet]. 2014 [cité 14 oct 2015]. Disponible sur: http://www.inserm.fr/thematiques/genetique-genomique-et-bioinformatique/dossiers-dinformation/drepanocytose

[17] Korb-Savoldelli V, Gillaizeau F, Pouchot J, Lenain E, Postel-Vinay N, Plouin PF, et al. Validation of a French Version of the 8-Item Morisky Medication Adherence Scale in Hypertensive Adults: Validation of a French Version of MMAS. J Clin Hypertens $2012 ; 14: 429 \square 34$.

[18] Kucukarslan SN. A review of published studies of patients' illness perceptions and medication adherence: Lessons learned and future directions. Res Soc Adm Pharm $2012 ; 8: 371 \square 82$.

[19] Lainé A, Dorie A. Perceptions de la drépanocytose dans les groupes atteints. In Bourse du travail de Seine St-Denis; 2009 [cité 21 oct 2015]. Disponible sur: https://hal.archivesouvertes.fr/hal-00432661/

[20] Lanzkron S, Haywood C, Segal JB, Dover GJ. Hospitalization rates and costs of care of patients with sickle-cell anemia in the state of Maryland in the era of hydroxyurea. Am J Hematol 2006;81:927 $\square 32$.

[21] Le Turdu-Chicot C, Foucan L, Etienne-Julan M, Leborgne-Samuel Y, Fanhan R, Berchel C. Analyse des hospitalisations chez les patients drépanocytaires adultes en Guadeloupe. Rev Médecine Interne 2000;21:25 $\square$.

[22] Leventhal H, Benyamini Y, Brownlee S, Diefenbach M, Leventhal EA, Patrick-Miller L, et al. Perceptions of Health and Illness: Theoritical foundations. In: Perceptions of health and illness: Current research and applications [Internet]. Harwood academic publisher; 1997 [cité 13 nov 2015]. p. $19 \square 45$.

[23] Lionnet F, Arlet J-B, Bartolucci P, Habibi A, Ribeil J-A, Stankovic K. Recommandations pratiques de prise en charge de la drépanocytose de l'adulte. Rev Médecine Interne 2009;30:S162 $\square 223$.

[24] Morisky DE, DiMatteo MR. Improving the measurement of self-reported medication nonadherence: Final response. J Clin Epidemiol 2011;64:262 \3.

[25] Morisky DE, DiMatteo MR. Improving the measurement of self-reported medication 


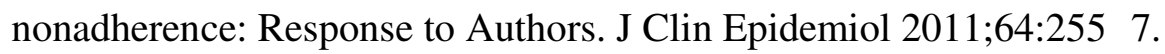

[26] Morisky DE, Ang A, Krousel-Wood M, Ward, HJ. Predictive Validity of A Medication Adherence Measure in an Outpatient Setting. J Clin Hypertens 2008;10:348 $\square 54$.

[27] Moss-Morris R, Weinman J, Petrie K, Horne R, Cameron L, Buick D. The Revised Illness Perception Questionnaire (IPQ-R). Psychol Health 2002;17:1 $\square 16$.

[28] Murray CJ, Barber RM, Foreman KJ, Ozgoren AA, Abd-Allah F, Abera SF, et al. Global, regional, and national disability-adjusted life years (DALYs) for 306 diseases and injuries and healthy life expectancy (HALE) for 188 countries, 1990-2013: quantifying the epidemiological transition. The Lancet 2015;386(10009):2145-91.

[29] Okam MM, Shaykevich S, Ebert BL, Zaslavsky AM, Ayanian JZ. National Trends in Hospitalizations for Sickle Cell Disease in the United States Following the FDA Approval of Hydroxyurea, 1998-2008: Med Care 2014;52:612 $\square$.

[30] OMS. The Ottawa Charter for Health Promotion [Internet]. 1986 [cité 21 déc 2016]. Disponible sur: http://www.who.int/healthpromotion/conferences/previous/ottawa/en/ [31] OMS. Aide-mémoire n³08 : Drépanocytose et autres hémoglobinopathies [Internet]. 2011 [cité 8 janv 2016]. Disponible sur: about:reader?url=http $\% 3 \mathrm{~A} \% 2 \mathrm{~F} \% 2 \mathrm{Fwww}$.who.int $\% 2 \mathrm{Fmediacentre} \% 2 \mathrm{Ffactsheets} \% 2 \mathrm{Ffs} 308 \% 2 \mathrm{Ff}$ $\mathrm{r} \% 2 \mathrm{~F}$

[32] Osakonor DK. Trait Anxiety in Sickle Cell Disease: investigating and exploring links to the management of pain [Internet]. University of East London; 2014 [cité 7 sept 2016]. Disponible sur: http://roar.uel.ac.uk/4628/

[33] Piel FB, Patil AP, Howes RE, Nyangiri OA, Gething PW, Dewi M, et al. Global epidemiology of sickle haemoglobin in neonates: a contemporary geostatistical model-based map and population estimates. The Lancet 2013;381(9861):142-51.

[34] Taylor LEV, Stotts NA, Humphreys J, Treadwell MJ, Miaskowski C. A Review of the Literature on the Multiple Dimensions of Chronic Pain in Adults with Sickle Cell Disease. J Pain Symptom Manage 2010;40:416 $\square 35$.

[35] Viswanathan K, Swaminathan N, Viswanathan R, Lakkaraja M. Caregiver's Health Locus of Control and Medication Adherence in Sickle Cell Disease. J Med Assoc 2015;107:51 $\square 5$.

[36] Voils CI, Hoyle RH, Thorpe CT, Maciejewski ML, Yancy Jr. WS. Improving the measurement of self-reported medication nonadherence: Response to Authors. J Clin Epidemiol 
$2011 ; 64: 258 \square 61$.

[37] Voils CI, Hoyle RH, Thorpe CT, Maciejewski ML, Yancy Jr. WS. Improving the measurement of self-reported medication nonadherence. J Clin Epidemiol 2011;64:250 4 .

[38] Wallen GR, Minniti CP, Krumlauf M, Eckes E, Allen D, Oguhebe A, et al. Sleep disturbance, depression and pain in adults with sickle cell disease. BMC Psychiatry 2014;14:1.

[39] Walsh KE, Cutrona SL, Kavanagh PL, Crosby LE, Malone C, Lobner K, et al. Medication Adherence Among Pediatric Patients With Sickle Cell Disease: A Systematic Review. Pediatrics 2014;134:1175 $\square 83$.

[40] Weinman J, Petrie KJ, Moss-Morris R, Horne R. The illness perception questionnaire: a new method for assessing the cognitive representation of illness. Psychol Health 1996;11:431-45. 
Tableau I: Synthèse des données socio-démographiques

$\mathbf{N}=79 \quad \% \quad \mu \pm \sigma \quad[\min ; \max ]$

\section{Phénotype}

$\begin{array}{rcc}\mathrm{SS} & 81,00 \% & 64 \\ \mathrm{SC} & 12,70 \% & 10 \\ \mathrm{~S} \beta^{+} & 3,80 \% & 3 \\ \text { Autre } & 2,50 \% & 2\end{array}$

Âge

$34,42 \pm 11,11$

$[16 ; 70]$

Lieux de naissance

$\begin{array}{rcc}\text { France et DOM/TOM } & 44,30 \% & 35 \\ \text { Afrique } & 51,90 \% & 41 \\ \text { Autre } & 3,80 \% & 3\end{array}$

\section{Niveau d'étude}

\begin{tabular}{|c|c|c|c|}
\hline Sans diplôme & $6,30 \%$ & & 5 \\
\hline BEPC / Brevet des collèges & $21,50 \%$ & & 17 \\
\hline Baccalauréat & $27,80 \%$ & & 22 \\
\hline $\mathrm{Bac}+1$ à +3 & $29,10 \%$ & & 23 \\
\hline Bac +4 et plus & $15,20 \%$ & & 12 \\
\hline Personnes en couple & $41,80 \%$ & & 33 \\
\hline \multicolumn{4}{|l|}{ Statut génétique du conjoint } \\
\hline Non porteur du gène & $71,00 \%$ & & 22 \\
\hline Hétérozygote (AS, AC, ...) & $12,90 \%$ & & 4 \\
\hline Homozygote (SS, SC, ... ) & $6,50 \%$ & & 2 \\
\hline «e ne sais pas » & $9,70 \%$ & & 3 \\
\hline Nombre d'enfant & & $1,05 \pm 1,23$ & {$[0 ; 6]$} \\
\hline Dont Homozygote (SS, SC, ...) & & $0,8 \pm 0,31$ & {$[0 ; 2]$} \\
\hline Dont Hétérozygote (AS, AC, ...) & & $0,52 \pm 0,98$ & {$[0 ; 5]$} \\
\hline
\end{tabular}

Lieu de diagnostic

$\begin{array}{rcc}\text { France } & 50,60 \% & 40 \\ \text { DOM/TOM } & 8,90 \% & 7 \\ \text { Afrique } & 35,40 \% & 28 \\ \text { Autre } & 5,10 \% & 4\end{array}$

Lieu de premier traitement

$\begin{array}{rcc}\text { France } & 53,20 \% & 42 \\ \text { DOM/TOM } & 6,30 \% & 5 \\ \text { Afrique } & 35,40 \% & 28\end{array}$


Autre

$5,10 \%$

4

Nombre d'hospitalisations

$3,33 \pm 3,90$

$[0 ; 20]$

Mesure subjective du bien-être

$6,46 \pm 2,46$

$[1 ; 10]$

$\mu$

$\pm$

$\sigma$

$\mathrm{m}$

o

y

e

n

$\mathrm{n}$

e

$\mathrm{t}$

é

c

a

$r$

$\mathrm{t}$

$\mathrm{t}$

$\mathrm{y}$ 
Tableau II: Résultats aux auto-questionnaires (IPQ-R, BMQ, MMAS).

\begin{tabular}{|c|c|c|}
\hline Questionnaires : & $\mu \pm \sigma$ & $\mathbf{N}$ \\
\hline \multicolumn{3}{|l|}{ IPQ-R (Illness Perception Questionnaire - Revised) } \\
\hline Identité & $5,83 \pm 2,64$ & 76 \\
\hline Chronologie & $23,71 \pm 4,92$ & 70 \\
\hline Conséquences & $20,81 \pm 4,76$ & 74 \\
\hline Contrôle Personnel & $21,84 \pm 4,10$ & 73 \\
\hline Contrôle Traitement & $17,67 \pm 3,44$ & 72 \\
\hline Cohérence perçue & $18,66 \pm 3,40$ & 76 \\
\hline Cyclicité & $14,17 \pm 2,97$ & 78 \\
\hline Représentation émotionnelle & $17,75 \pm 5,92$ & 71 \\
\hline Cause : Hérédité/Génétique & $15,81 \pm 2,58$ & 75 \\
\hline Cause : Origine géographique & $10,23 \pm 2,42$ & 73 \\
\hline Cause : Sang & $16,56 \pm 3,37$ & 71 \\
\hline Cause : Magie & $6,74 \pm 2,34$ & 77 \\
\hline Cause : Comportement Actif & $9,97 \pm 3,95$ & 76 \\
\hline Cause : Comportement Passif & $10,93 \pm 4,49$ & 77 \\
\hline \multicolumn{3}{|c|}{ BMQ (Beliefs about Medecine Questionnaire) } \\
\hline Nécessité & $17,56 \pm 5,00$ & 77 \\
\hline Préoccupations & $13,91 \pm 4,39$ & 76 \\
\hline Nuisance & $10,38 \pm 3,24$ & 79 \\
\hline Abus & $10,42 \pm 3,14$ & 74 \\
\hline Nécessité - Préoccupations & $3,77 \pm 5,89$ & 74 \\
\hline MMAS (Morisky Medication Adherence Scale) & $4,04 \pm 1,81$ & 62 \\
\hline
\end{tabular}


Tableau III: Corrélations des dimensions de la représentation (IPQ-R) avec les dimensions de l'adhésion (BMQ) et l'observance thérapeutique (MMAS).

\begin{tabular}{|c|c|c|c|c|c|c|}
\hline VARIABLE & Nécessité & Préoccupations & Nuisance & Abus & $\begin{array}{c}\text { Différence } \\
\text { Nécessité- } \\
\text { Préoccupations }\end{array}$ & MMAS \\
\hline Identité & $\begin{array}{l}, 10 \\
(, 39)\end{array}$ & $\begin{array}{l}, 16 \\
(, 16)\end{array}$ & $\begin{array}{l}-, 08 \\
(, 51)\end{array}$ & $\begin{array}{l}-, 03 \\
(, 80)\end{array}$ & $\begin{array}{l}-, 03 \\
(, 82)\end{array}$ & $\begin{array}{l}-, 20 \\
(, 12)\end{array}$ \\
\hline Durée & $\begin{array}{l}, 08 \\
(, 53)\end{array}$ & $\begin{array}{l}-, 04 \\
(, 76)\end{array}$ & $\begin{array}{l}-, 10 \\
(, 39)\end{array}$ & $\begin{array}{l}-, 21 \\
(, 08)\end{array}$ & $\begin{array}{l}, 11 \\
(, 37)\end{array}$ & $\begin{array}{l}, 25 \\
(, 07)\end{array}$ \\
\hline Conséquences & $\begin{array}{l}, 16 \\
(, 18)\end{array}$ & $\begin{array}{l}, 40^{* *} \\
(, 00)\end{array}$ & $\begin{array}{l}-, 02 \\
(, 89)\end{array}$ & $\begin{array}{c}, 04 \\
(, 71)\end{array}$ & $\begin{array}{c}-0,21 \\
(, 08)\end{array}$ & $\begin{array}{c}-, 28^{*} \\
(, 03)\end{array}$ \\
\hline Contrôle personnel & $\begin{array}{l}, 09 \\
(, 44)\end{array}$ & $\begin{array}{l}, 06 \\
(, 61)\end{array}$ & $\begin{array}{l}-, 17 \\
(, 15)\end{array}$ & $\begin{array}{l}-, 22 \\
(, 06)\end{array}$ & $\begin{array}{l}, 08 \\
(, 51)\end{array}$ & $\begin{array}{c}, 07 \\
(, 61)\end{array}$ \\
\hline Contrôle traitement & $\begin{array}{c}\mathbf{3 7 5}^{* *} \\
(, 001)\end{array}$ & $\begin{array}{l}-, 05 \\
(, 70)\end{array}$ & $\begin{array}{l}-, 09 \\
(, 43)\end{array}$ & $\begin{array}{c}-, 30^{*} \\
(, 01) \\
\end{array}$ & $\begin{array}{l}, 37^{* * *} \\
(, 002)\end{array}$ & $\begin{array}{l}\text {,29* } \\
(, 03) \\
\end{array}$ \\
\hline Cohérence & $\begin{array}{l}, 11 \\
(, 35)\end{array}$ & $\begin{array}{l}-, 20 \\
(, 09)\end{array}$ & $\begin{array}{l}-, 09 \\
(, 42)\end{array}$ & $\begin{array}{c}-, 45^{* *} \\
(, 00)\end{array}$ & $\begin{array}{l}\text {,28* } \\
(, 02)\end{array}$ & $\begin{array}{l}, 21 \\
(, 10)\end{array}$ \\
\hline Cyclicité & $\begin{array}{c}, 09 \\
(, 46)\end{array}$ & $\begin{array}{l}, 15 \\
(, 20)\end{array}$ & $\begin{array}{l}-, 02 \\
(, 85)\end{array}$ & $\begin{array}{l}-, 05 \\
(, 69)\end{array}$ & $\begin{array}{l}-, 07 \\
(, 57)\end{array}$ & $\begin{array}{l}-, 24 \\
(, 06)\end{array}$ \\
\hline Représentation émotionnelle & $\begin{array}{l}\mathbf{3 2}^{* *} \\
(, 007)\end{array}$ & $\begin{array}{l}, 44^{* *} \\
(, 00)\end{array}$ & $\begin{array}{c}, 07 \\
(, 56)\end{array}$ & $\begin{array}{l}-, 12 \\
(, 30)\end{array}$ & $\begin{array}{c}-, 1 \\
(, 43)\end{array}$ & $\begin{array}{l}-, 21 \\
(, 12)\end{array}$ \\
\hline Cause : Hérédité/Génétique & $\begin{array}{l}27^{*} \\
(0,2)\end{array}$ & $\begin{array}{l}, 10 \\
(, 39)\end{array}$ & $\begin{array}{c}, 13 \\
(, 25)\end{array}$ & $\begin{array}{l}\text {,01 } \\
(, 91)\end{array}$ & $\begin{array}{c}, 12 \\
(, 31)\end{array}$ & $\begin{array}{c}, 12 \\
(, 36)\end{array}$ \\
\hline Cause : Origine géographique & $\begin{array}{c}0,6 \\
(, 59)\end{array}$ & $\begin{array}{l}, 09 \\
(, 45)\end{array}$ & $\begin{array}{l}, 25^{*} \\
(, 03)\end{array}$ & $\begin{array}{l}, 22 \\
(, 06)\end{array}$ & $\begin{array}{l}-, 04 \\
(, 74)\end{array}$ & $\begin{array}{l}-, 19 \\
(, 15)\end{array}$ \\
\hline Cause : Sang & $\begin{array}{l}, 06 \\
(, 62)\end{array}$ & $\begin{array}{l}-, 02 \\
(, 87)\end{array}$ & $\begin{array}{l}, 09 \\
(, 46)\end{array}$ & $\begin{array}{l}, 08 \\
(, 53)\end{array}$ & $\begin{array}{c}, 04 \\
(, 74)\end{array}$ & $\begin{array}{l}-, 13 \\
(, 34)\end{array}$ \\
\hline Cause : Magico-religieuse & $\begin{array}{l}-, 20 \\
(, 08)\end{array}$ & $\begin{array}{l}-, 02 \\
(, 88)\end{array}$ & $\begin{array}{l}, 18 \\
(, 12)\end{array}$ & $\begin{array}{l}, 32^{* *} \\
(, 004)\end{array}$ & $\begin{array}{l}-, 20 \\
(, 08)\end{array}$ & $\begin{array}{l}-, 35^{* *} \\
(, 005)\end{array}$ \\
\hline Cause : Comportement actif & ,03 &,- 12 &, 11 & ,03 &, 11 &,- 17 \\
\hline
\end{tabular}




\begin{tabular}{|c|c|c|c|c|c|c|}
\hline VARIABLE & Nécessité & Préoccupations & Nuisance & Abus & $\begin{array}{c}\text { Différence } \\
\text { Nécessité- } \\
\text { Préoccupations }\end{array}$ & MMAS \\
\hline & $(, 78)$ & $(, 30)$ & $(, 36)$ & $(, 82)$ & $(, 35)$ & $(, 18)$ \\
\hline Cause : Comportement passif & $\begin{array}{c}, 06 \\
(, 63)\end{array}$ & $\begin{array}{c}, 03 \\
(, 80)\end{array}$ & $\begin{array}{l}\mathbf{3 0}^{* *} \\
(, 007)\end{array}$ & $\begin{array}{l}, 14 \\
(, 22)\end{array}$ & $\begin{array}{c}, 01 \\
(, 90)\end{array}$ & $\begin{array}{l}-,, 31 * \\
(, 014)\end{array}$ \\
\hline
\end{tabular}

$*: p<0,05 ; * *: p<0,01$ 
Tableau IV: Taux d'acceptation des causes (IPQ-R)

\section{Causes perçues de la drépanocytose}

$\mathbf{n}=$

1 Cause : Sang

43

42

$60,6 \%$

2 Cause : Hérédité/Génétique

$9 \quad 12,3 \%$

3 Cause : Origine géographique

$4 \quad 5,2 \%$

4 Cause : Comportement passif

$1 \quad 1,3 \%$

6 Cause : Magico-religieuse

$1 \quad 1,3 \%$


Tableau V: Prédicteurs de l'influence de la représentation de la maladie sur l'observance thérapeutique et sur l'adhésion thérapeutique - régression linéaire.

Régression sur l'indicateur d'observance thérapeutique (MMAS)

\begin{tabular}{lccc} 
& $\beta$ & Erreur Standad & $\mathrm{t}$ \\
\cline { 2 - 4 } Constante & & 1,96 &,- 06 \\
Représentation émotionnelle &,- 32 &, 04 & $-2,51^{*}$ \\
Contrôle traitement &, 34 &, 07 & $2,66^{*}$ \\
Chronologie &, 31 &, 05 & $2,45^{*}$ \\
SCT $=144$ & & & \\
$\mathrm{R}^{2}=, 29$ & & &
\end{tabular}

Régression sur l'indicateur d'adhésion thérapeutique (différence Nécessité-Préoccupations)

\begin{tabular}{lccc} 
& $\beta$ & Erreur Standad & $\mathrm{t}$ \\
\cline { 2 - 4 } Constante & & 1,84 &,- 11 \\
Représentation émotionnelle &,- 36 &, 04 & $-2,84^{* *}$ \\
Contrôle traitement &, 37 &, 06 & $2,91^{* *}$ \\
Chronologie &, 36 &, 05 & $2,79^{* *}$ \\
SCT $=144$ & & & \\
$\mathrm{R}^{2}=, 34$ & & & \\
$* *: p<0,01 ; *: p<0,05$ & & &
\end{tabular}

